\title{
The Perioperative Quality Improvement Programme (PQIP Patient Study): Protocol for a UK Multicentre, Prospective Cohort Study to Measure, Report and Improve Quality and Outcomes of Major Surgery
}

\section{S. Ramani Moonesinghe ( $\square$ rmoonesinghe@gmail.com )}

University College London Hospitals NHS Foundation Trust https://orcid.org/0000-0002-6730-5824

Dermot McGuckin

UCL: University College London

Peter Martin

UCL: University College London

James Bedford

UCL: University College London

Duncan Wagstaff

UCL: University College London

Cecilia Vindrola-Padros

UCL: University College London

Arun Sahni

UCL: University College London

Kylie Edwards

UCL: University College London

Georgina Singleton

UCL: University College London

Pritam Singh

Nottingham City Hospital

R.Jonathan T. Wilson

York University Hospital

David Anthony Gilhooly

UCL: University College London

Rachel Baumber

UCL: University College London

Anne-Marie Bougeard

Torbay and South Devon NHS Foundation Trust

Katie Samuel

North Bristol NHS Trust 


\section{Alexandra Brent}

Health Services Research Centre

Maria Chazapis

University College London Hospitals NHS Foundation Trust

Martin Cripps

Netsolving Ltd

Jenny Dorey

Royal College of Anaesthetists

Irene Leeman

Royal College of Anaesthetists

Helena Smith

University College London Hospitals NHS Foundation Trust

\section{Michael Swart}

Torbay and South Devon NHS Foundation Trust

\section{Sharon Drake}

Royal College of Anaesthetists

\section{James Goodwin}

Royal College of Anaesthetists

Jose Lourtie

Royal College of Anaesthetists

\section{Dorian Martinez}

Royal College Of Anaesthetists

Dominic Olive

Royal College of Anaesthetists

\section{Christine Taylor}

Royal College of Anaesthetists

\section{Karen Williams}

Royal College of Anaesthetists

\section{Cristel Santos}

Royal College of Anaesthetists

\section{Helen Ellicott}

Health Services Research Centre

\section{Samantha Warnakulasuriya}

University College London Hospitals NHS Foundation Trust

\section{Aleksandra Ignacka}

Health Services Research Centre

\section{Matthew Bedford}

University Hospitals Birmingham NHS Foundation Trust

\section{Giuseppe Aresu}


Papworth Hospital NHS Foundation Trust: Royal Papworth Hospital NHS Foundation Trust

\section{Olga Tucker}

University Hospitals Birmingham NHS Foundation Trust

\section{Abigail E Vallance}

Bristol University

\section{Ravinder Vohra}

Nottingham University Hospitals NHS Trust

\section{Andrew Swift}

Aintree University Hospitals NHS Foundation Trust: Liverpool University Hospitals NHS Foundation Trust Naomi J Fulop

University College London

Michael PW Grocott

University of Southampton

\section{Methodology}

Keywords: Perioperative Quality Improvement Programme (PQIP), Major surgery, UK multicentre, cohort study, improve quality, patient study, protocol

Posted Date: July 14th, 2021

DOI: https://doi.org/10.21203/rs.3.rs-708161/v1

License: (c) (i) This work is licensed under a Creative Commons Attribution 4.0 International License. Read Full License 


\section{Abstract}

\section{Background}

Major surgery accounts for a substantial proportion of health service activity and resource consumption, due not only to the primary procedure, but also the short and long-term implications of perioperative complications. It is likely that both compliance with best practice processes and outcomes from major surgery vary substantially between hospitals and therefore could be targets for quality improvement.

\section{Methods}

The Perioperative Quality Improvement Programme (PQIP) patient study is a multi-centre prospective cohort study which recruits participants undergoing major inpatient non-cardiac surgery with three main aims: to measure and improve processes of care and outcome from major surgery; to implement and evaluate a complex intervention aiming to enhance the use of data for improvement by clinical teams; and to create a national database to support collaborative research and efficient study design. The prospective dataset combines variables for risk adjustment, process measures and both objective and patient reported outcome data. Longer-term outcomes are collected through linkage to national administrative datasets (mortality and readmissions). PQIP deploys a theoretically underpinned improvement methodology to support the use of data for improvement by perioperative clinicians, incorporating action research principles to enable changes to be made in response to user feedback. Dissemination of interim findings (non-inferential) form a part of the improvement methodology and are provided to participating centres at regular intervals, including near-real-time feedback of key process measures. Inferential analyses will be published in the peer-reviewed literature, supported by a multimodal communications strategy to patients, public, policy makers and academic audiences as well as clinicians.

\section{Discussion}

PQIP is the first national effort in the UK to measure and report risk-adjusted complications, patientreported outcome and mortality rates for patients undergoing major surgery across multiple surgical specialties in the UK. Its main limitation is the risk of sampling bias due to the requirement for patient consent, and because local resource constraints may lead hospitals to recruit a convenience sample, rather than a truly random sample. We will evaluate this risk by using Hospital Episode Statistics (HES) to identify all patients undergoing PQIP eligible procedures, and undertaking sensitivity analyses comparing common data points in the PQIP sample and the HES population. As the purpose of PQIP is to support quality improvement and research as opposed to quality assurance or institutional comparisons, even if they exist, such sampling biases are unlikely to materially affect the ability of the programme to achieve its aims. 


\section{Introduction}

Approximately 10 million procedures are carried out in the UK National Health Service (NHS) each year, of which 1.5 million would be classed as 'major surgery'. ${ }^{1}$ Globally, surgical caseload substantially exceeds 300 million procedures per annum. ${ }^{2}$ International estimates of death within 30 days of planned surgery vary, but it is acknowledged to be relatively uncommon in high income nations (around $0.6-4 \%$ depending on the study design, health system and population characteristics). ${ }^{134}$ However, there are several reasons why these low estimates of short-term mortality may provide false reassurance about the quality of perioperative care and the potential health and economic burden on patients and society. First, the incidence of major or prolonged postoperative morbidity is at least ten times higher than short-term mortality. ${ }^{5}$ This is important, because major morbidity contributes to increased length of hospital stay and associated increased cost of healthcare. Second, US data have shown wide variation in risk-adjusted morbidity and failure-to-rescue rates between healthcare providers, suggesting that at least some of these poor short-term outcomes could be avoidable. ${ }^{4,6}$ However, the lack of a unified NHS system for measuring complications across different types of major surgery means that it is currently not possible to ascertain if the UK has the same problem. Third, a consistent finding across different procedures, populations and healthcare systems is the independent association between short-term postoperative complications and reduced longer-term survival. ${ }^{5,78}$. It therefore follows, that the outcome of surgery should not be defined entirely by short-term survivorship. Longer-term and additional patient-centred outcomes such as quality of life or disability-free survival are increasingly viewed as important measures of perioperative 'success' by both clinicians and patients, but there is comparatively little high quality data on these outcomes. ${ }^{9,10}$ This is particularly important given the evolving demographics of the surgical population (increasing age and multi-morbidity) and the wider availability of surgical options for previously untreatable conditions. There is also a recognition that not all interventions are in the best interests of patients, and sometimes less invasive approaches may lead to better outcomes and fewer adverse events. ${ }^{11} 12$ Thus, understanding the full implications of a surgical intervention is critical to supporting high quality patient care and shared decision making.

Beyond understanding these issues more clearly, there is also a need to measure and improve structures and processes associated with perioperative care, so that outcomes may also improve. Numerous national audit and quality improvement initiatives are already operational in the UK, ranging from shortterm evaluations of specific clinical areas (e.g. National Confidential Enquiry into Patient Outcome and Death (NCEPOD)) to longer-term continuous national registries and audits (e.g. the National Joint Registry or the National Emergency Laparotomy Audit (NELA)). While these audits are important for national monitoring and quality assurance, the lag time between data collection and publication of results can be a significant barrier to quality improvement (QI) which requires sustainable prospective data collection and real-time presentation and clinician use of results. Thus, the use of data from these programmes to drive local QI activity varies considerably. This variation is also related to aspects of programme delivery as well as to local factors related to culture, resources and clinician engagement. ${ }^{13}$ 
The PQIP patient study attempts to address these areas of need through a comprehensive national programme of data collection across multiple surgical specialties and feedback, incorporating a multilevel complex intervention aimed at supporting the use of data for improvement at local level. We describe here the protocol for the study.

\section{Methods}

\section{Main study design}

Prospective cohort study of patients undergoing major surgery in NHS hospitals. Observational study at patient level; interventional action research study using time-series analysis at hospital level.

\section{Aims}

A1. To measure and improve outcome from major surgery in the UK NHS.

A2. To implement and evaluate a complex intervention aiming to enhance the use of data for improvement by clinical teams

A3. To create a national resource for the collection, management and analysis of high-quality perioperative data, to support collaborative research and efficient study design.

\section{Objectives}

Obj 1. To comprehensively measure and report structures, processes and risk-adjusted complications, patient-reported outcome and mortality rates after major surgery

Obj 2. To evaluate the relationships between structures, processes and outcomes after major surgery

Obj 3. To develop bespoke, regularly updated and non-proprietary risk models for the purposes of casemix adjustment and perioperative risk prediction for different outcomes

Obj 4. To support local quality improvement through feedback of data to clinicians and managers using near-real-time feedback and regular comprehensive reporting.

Obj 5. To develop and evaluate the effectiveness of a theoretically underpinned complex intervention involving novel methods of data feedback, analysis and support mechanisms to aid local improvement initiatives.

Obj 6. To support the development and delivery of collaborative nested studies

\section{Research Questions}

RQ1. What is the level of compliance with evidence-based structures and processes for patients undergoing major surgery? 
RQ2. What are the rates and predictors of complications, failure to rescue (FTR, defined as mortality within 30 days occurring after the presence of morbidity on postoperative day 7), prolonged length of stay, short- and long- term mortality in patients undergoing major planned surgery in NHS hospitals?

RQ3. How do the rates of adverse outcomes vary over time and between institutions?

RQ4. What are the structural or process related predictors of these adverse outcomes?

RQ5. What is the relationship between different measures of postoperative outcomes (e.g. different measures of morbidity; short and longer-term outcomes, health-related quality of life and disability)?

RQ6. What is the validity and predictive performance of previously published risk prediction models for different perioperative outcomes in the PQIP population?

RQ7. Can existing risk models be improved, or more parsimonious versions developed to support temporally responsive risk adjustment and clinically useful risk evaluation?

RQ8. How representative is the sample of patients recruited to PQIP of the total cohort of patients undergoing potentially eligible procedures in PQIP hospitals?

RQ9. Is there an outcome benefit to hospital and/or patient involvement in PQIP which can be differentiated from secular variation?

RQ10. Can quality of care be improved through a theoretically underpinned complex intervention supporting the use of data for improvement at local level, and do patient outcomes also improve?

\section{Ethics}

The study has been approved by the Health Research Authority (HRA) as a research study, following review at the South-East Coast - Surrey Research Ethics Committee (REC reference: 16/LO/1827; IRAS project ID: 215928). All hospitals provide Caldicott Guardian approval in addition to confirmation of capacity and capability in accordance with usual HRA requirements.

\section{Participants}

All NHS hospitals which undertake any of the included procedures are eligible to participate. Hospitals are approached via the Royal College of Anaesthetists and its Health Services Research Centre, and via the National Institute for Health Research's Clinical Research Networks. Patients aged 18 years or older undergoing planned major surgery (as defined by a list of index procedures requiring inpatient stay) are eligible for recruitment and patient consent is sought pre-operatively. Patient consent is required as the study seeks additional data to that collected as part of routine care (specifically the patient-reported outcome data). The consenting process is undertaken by staff locally trained and approved. The participant information provides detail on how data are collected, the request for patient reported data, and the linkage of hospital data with external databases (see below, dataset). 
Hospitals may choose to recruit patients from all specialties or specify particular specialties where they wish to focus at local level. Each hospital is offered the opportunity to either approach all eligible patients for consent, or all patients within a particular specialty or plan to recruit between one and five patients per week, approaching patients for written consent based on a random sampling strategy. This will involve an eight-day rolling sampling cycle (i.e. for week one the first five patients starting on Monday morning, followed by the first five patients starting from Tuesday in week two, et cetera). If any of the first five patients approached decline to consent, then consecutive patients will be approached for consent until the target recruitment number has been achieved. Patients can choose to be withdrawn from the study at any time.

\section{Sample size}

Target recruitment is 70,000 patients. It is expected that this will take at least 5 years to achieve. The long observation period will facilitate the study of how data collection and feedback impacts upon patient outcomes over time.

\section{Dataset}

The dataset has been informed using the best available evidence. Recorded outcomes include: postoperative morbidity defined using the Post-Operative Morbidity Survey, collected on day 7 after surgery; ${ }^{14}$ POMS-defined major morbidity; ${ }^{15}$ complications graded according to the Clavien-Dindo classification; ${ }^{16}$ failure to rescue (FTR); ${ }^{1718}$ resource utilisation (critical care admission (planned/unplanned); critical care length of stay, hospital length of stay, hospital readmission within 30 days of index procedure); mortality at 90 days; days alive and out of hospital ${ }^{1920}$ censored at 30days, 60 days, 90 days and one year postoperatively; disability-free survival at one year; change in patient reported health related quality of life (HRQOL) between baseline and 6 and 12 months after surgery; the responses to the "Ask2Questions" brief questionnaire about complex pain. ${ }^{21}$ The choice of outcome measures has been based on formal validation studies ${ }^{14,22,23}$ and expert consensus. ${ }^{20,24-30}$ To calculate FTR, we will follow previously described methodology, but using the presence of a Clavien-Dindo grade II or above complication as our definition of postoperative complications. ${ }^{17,18}$ Candidate variables for risk adjustment were selected from the results of a systematic review, and subsequent original research ${ }^{31-33}$. Structure and process measures have been informed by systematic review ${ }^{34}$ and Delphi consensus process. An expert panel (the Clinical Reference Group) of stakeholders from different medical and surgical specialties provided further input to refine the long-list of potential measures. In addition to prospectively collected data, we will also link patient-level data with NHS Digital Hospital Episode Statistics and the Office of National Statistics mortality register. We will also consider patient-level linkage with other registries such as the National Cancer Registry, the Intensive Care National Audit and Research Centre's Case-Mix Programme and relevant National Clinical Audits, in order to provide a comprehensive dataset at lowest local data collection burden.

\section{Study flow}


Patients are approached for participation either by mail, at the preoperative assessment clinic or on the day of surgery. A minimum of 1 hour is allowed for patients to consider the information on the participant information sheet before they are asked whether they wish to consent. At the time of consent, patients are asked to indicate whether they wish their longer-term follow-up to be by email or telephone. Following consent, the patient is asked to complete baseline demographic and HRQOL data. On the day of surgery, clinical teams or research staff complete preoperative, intraoperative and recovery room data. Further objective data capture occurs on day 2 or 3 (the Drinking Eating and Mobilising process measure ${ }^{35}$ ), day 7 (the Post-Operative Morbidity Survey (POMS) ${ }^{14}$ ) and at hospital discharge (the Clavien-Dindo complications grading scale, ${ }^{16,36}$ and information about the length of stay and post-discharge destination of the patient. In addition, patients are asked to complete a patient satisfaction with anaesthesia questionnaire 37,38 within $24 \mathrm{~h}$ of surgery, a quality of recovery questionnaire at baseline (at the time of consenting to participate in PQIP) and postoperative day $3,39,40$ and longer-term HRQOL and disability-assessment questionnaires at baseline, 6 and 12 months after surgery. ${ }^{2223}$.

\section{Loss to follow-up}

We are conscious that with a large pragmatic study of this nature without substantial funding but with multiple follow-up points and hospitals participating, that there is the potential for significant loss to follow-up. We have considered this as follows:

Inpatient morbidity and complication follow-up rates are predicted to be high, as they are collected inhospital. We have created logic checks within our online data entry tool to enable us to differentiate between missing data and negative responses. Hospitals with large amounts of missing data will be contacted and offered support.

Mortality follow-up rates are likely to be high, as availability of the information does not depend on patients being contactable or willing to respond to a questionnaire (through linkage with NHS databases). There will be a small number of patients for whom data linkage is not possible (for example if they have left the NHS) but we will receive information about this type of loss to follow-up from NHS Digital.

For longer-term patient-reported outcomes, we will take measures to maximise response rates, for example through multiple reminder letters \& phone calls. Additionally, and where required and appropriate, we will conduct sensitivity analyses to understand if there are any important differences between responders and non-responders and to assess for potential biases

\section{Co-enrolment}

There is no barrier to PQIP patients being co-enrolled into other studies. We actively seek collaboration with other researchers to facilitate co-enrolment with other studies while paying attention to the need to minimise redundant data collection and data collection fatigue of patients and investigators.

\section{Analysis Plan}




\section{Descriptive statistics (RQ1, RQ2)}

Descriptive statistics will be used to describe the basic demographics of participants. We will report process measures of engagement with PQIP (case-ascertainment rates, data completion) and compliance with processes of care at local and national level. (RQ1) Both unadjusted and adjusted outcomes will be reported (see inferential statistics for details of risk adjustment). (RQ2) All hospital level data will be presented anonymously.

\section{Missing Data}

For each statistical analysis, we will document the number and rate of missing observations on all variables involved. For each statistical model, we will assess the likely process that lead to missing observations, and whether data are likely to be missing at random (MAR) or missing not at random (MNAR). This assessment will inform a decision about the appropriate method of analysis. For example, multiple imputation or imputation of normal values may be considered for predictors in statistical models, depending on the likely process of missingness. Patients who are missing outcome data will be omitted from the relevant analysis; sensitivity analyses will be conducted to evaluate differences between patients with and without missing outcome data.

\section{Temporal and between-hospital variation in outcomes (RQ3)}

We will assess the variation over time and between hospitals on all primary and secondary outcome measures. Risk-adjustment will be based on logistic regression or other regression models, as appropriate for each outcome measure. Both patient-level and operation-level predictors will be included, such as age, gender, operation type and the constituent variables of previously published and validated risk adjustment models such as the Portsmouth Physiological and Operative Score for the enumeration of morbidity and mortality (P-POSSUM) and the Surgical Outcome Risk Tool (SORT) ${ }^{41 ;}{ }^{32}$.

\section{Association between outcomes and hospital structures and processes of care (RQ4)}

To describe the relationship between outcomes and hospital structures and processes, we will use mixedeffects models, employing random coefficients to assess the variation between hospitals. Patient-level covariates will be included as appropriate to distinguish the effect of case-mix from the effect of hospital characteristics on outcomes.

\section{Relationship between different postoperative outcomes (RQ5)}

We will evaluate the association between different outcome measures which purport to assess similar constructs (e.g. short-term morbidity) and between outcomes which may be associated with each other. As an example for the latter, the relationship between short-term complications and long-term survival has been demonstrated previously; ${ }^{578}$ however, there are fewer data evaluating the relationship between a complicated postoperative course and health-related quality of life or disability free survival. We will use odds ratios, Pearson correlations and statistical measures of agreement as appropriate. 


\section{Evaluation of existing risk prediction models (RQ 6)}

We will evaluate discrimination (using the area under receiver-operator-characteristics curves) and calibration (using Hosmer-Lemeshow or Pearson correlation statistics) of known risk prediction models including the P-POSSUM, SORT and Surgical Risk Scale.

\section{Developing new models for the prediction of risk (RQ 7)}

We will develop and internally validate new models for the prediction of risk, using logistic or other regression models as appropriate. Penalised regression models will be considered to reduce the risk of overfitting. These analyses may lead to a modification of the dataset with the aim of reducing data collection burden, if we find parsimonious models that are able to do without some variables previously considered important for risk prediction.

\section{Evaluating sample validity (RQ8)}

One of the objectives of PQIP is to measure and report processes and outcomes from surgery for the purposes of quality improvement. While it is widely accepted that data from a non-random sample of patients within an institution may provide important information about reliability of processes and systems, ${ }^{42}$ sampling of patient outcomes should be statistically and conceptually robust so as not to provide biased estimates. Clinical trials and cohort studies which have specific inclusion and exclusion criteria, or which require patient consent, can be criticised as non-representative (and therefore the findings are of limited generalisability). ${ }^{43} \mathrm{~A}$ potential solution to this is to use administrative data to evaluate outcomes; however, in the UK, such data are limited to mortality, hospital readmission and length of stay.

We will address these issues through an evaluation of our sampling strategy using administrative data from Hospital Episode Statistics (HES). For included procedures, an anonymised extract of HES data will be requested from NHS Digital for each hospital. This will enable us to conduct sensitivity analyses comparing patient characteristics (for example age, comorbidity, socioeconomic status using the Index of Multiple Deprivation) and outcomes (mortality, hospital readmission, length of hospital stay) between patients included in PQIP and those who are not. We will do this at individual hospital level and at aggregate (national) level.

\section{Evaluating impact of PQIP (RQ9)}

We will compare risk-adjusted patient outcomes which are available from administrative data (e.g. mortality, length of stay, readmission to hospital, 'happy days' censored at 90 days and one year after surgery) in hospitals and patients who are enrolled in PQIP and those who are not. Temporal trends will be analysed using a difference in differences approach. In order to avoid bias associated with different hospital types which may affect patient outcomes irrespective of involvement with PQIP, we will apply coarsened exact matching, ${ }^{44,45}$ matching PQIP and non-PQIP hospitals using pooled data from 
organisational surveys conducted by the Health Services Research Centre for PQIP and other studies (e.g. SNAP2-EPICCS ${ }^{46}$ and the National Emergency Laparotomy Audit ${ }^{47}$ ) as well as open-access external sources such as NHS England and Department of Health datasets.

\section{Embedded further research}

\section{PQIP evaluation (RQ10)}

One of the core aims of PQIP is to enhance the use of data for improvement by clinical teams, and in so doing, improve processes and outcomes for patients. PQIP may be considered as a complex intervention, which has been developed based on two theoretical frameworks (Normalisation Process Theory 48, 49 and the Theoretical Domains Framework ${ }^{50}$ ). The intervention involves every part of the measurement, audit and feedback cycle and beyond, and includes collaborative events, regular reporting, dashboards of key measures and the use of social media and educational media to disseminate knowledge and learning. We are evaluating the effectiveness and processes of implementation of PQIP (RQ9) using both qualitative and quantitative methods. Quantitative methods will include the temporal analysis described to address RQs 3 and 9, and longitudinal survey work which will evaluate engagement with the use of PQIP data. The qualitative research will take the form of a multi-sited ethnography, using interviews and observations with frontline staff across PQIP and non-PQIP sites and PQIP project team members. The protocol for this evaluation and a description of the development of the intervention will be published separately.

\section{Nested studies and secondary use of PQIP data}

The multi-centre nature of PQIP provides an opportunity to develop and conduct multiple further studies in sub-groups of patients or hospitals, to pilot and evaluate new interventions, and then consider wider implementation. Such interventions may be aimed at improving processes and outcomes of care for patients (core aim 1 of PQIP) or improving the use of data for improvement (core aim 2 of PQIP). Two examples are briefly outlined below. In addition, PQIP has the potential to be used as a clinical trials platform, investigating new treatments or pathways using a randomised design. The PQIP project team will continue to consider requests from external clinicians and researchers collaborate and modify the PQIP dataset to maximise its usefulness for QI or research. After our planned analyses have been completed, a process will be established to enable external clinicians and researchers to apply for access to an anonymised dataset to explore research hypotheses.

\section{pomVLAD}

Post-Operative Morbidity reporting using Variable Life Adjusted Displays (pomVLAD), is a nested study funded by the Health Foundation which will develop, implement and evaluate the effectiveness of a near real-time reporting system for risk-adjusted morbidity, mortality and FTR rates. Originally developed to monitor observed versus expected mortality in cardiac surgery, VLAD provides a graphical display of riskadjusted outcome data over time. ${ }^{51}$ We plan to develop an recommendation bundle which will be paired 
with the VLAD display. The enhanced recovery bundle and VLAD dashboard will be trialled in ten early adopter hospitals and evaluated using a difference-in-differences analysis to evaluate its effectiveness and feasibility 52 .

\section{ERAS Plus}

The ERASPlus initiative (erasplus.co.uk) is an expansion of a quality improvement programme aimed at reducing postoperative pulmonary complications. It was initially implemented, evaluated and found to be clinically effective in a single centre ${ }^{53}$ and is now being rolled out throughout the Greater Manchester area. The intervention consists of a bundle of care supported by technological innovation (an app for patients to use) including exercise advice and training and targeted interventions to improve pulmonary function and reduce the risk of complications. The expanded ERASPlus initiative is also being funded by the Health Foundation and is working with PQIP to combine data collection efforts to reduce burden on local teams; additionally, this will facilitate comparisons with the non-ERAS+ population of PQIP patients.

\section{Data management and linkage}

All investigators and study site staff comply with the requirements of the Data Protection Act 1998 with regards to the collection, storage, processing and disclosure of personal information and will uphold the Act's core principles. Patient-level data is entered by local investigators into a secure, electronic, webbased database. This is hosted on servers managed by UK Fast on behalf of the RCoA. Local investigators have access to their own full datasets. An anonymised dataset will be used by the PQIP Project Team for analysis. In this dataset: NHS number is replaced by a unique study patient identifier; date of birth is converted to age on date of surgery; postcode is converted to primary care trust (PCT), strategic health authority (SHA) of residence and the ONS Lower Super Output Area to allow the allocation of the Index of Multiple Deprivation.

The minimum amount of patient identifiable data (including PQIP unique identifier, NHS number, date of birth, gender and postcode) will be extracted from the study database by the PQIP Project Team to allow data linkage to ONS mortality data and Hospital Episode Statistics data from NHS Digital, so that we may track post-discharge outcomes (e.g. hospital readmission) and long-term survival.

\section{Data sharing}

Local data can be exported by approved local investigators at each hospital at any time. We will invite PQIP collaborators to apply for multi-centre data to undertake their own secondary analyses which are outside our initial analysis plans. These applications will be reviewed by the PQIP project team and assistance offered for analysis and interpretation, subject to capacity. Applications for access to PQIP data by non-PQIP collaborators will be considered when the study and the planned analyses are completed. Fully anonymised datasets will be made available on data sharing resources once the study and all follow-up is complete. 


\section{Patient and public involvement}

The PQIP patient study addresses areas prioritised by four James Lind Alliance Priority Setting Partnerships (JLA-PSPs): anaesthesia/perioperative care, intensive care, dementia \& pressure ulcers. We received detailed structured feedback on our protocol from members of the PCPIE group at the National Institute for Academic Anaesthesia's Health Services Research Centre (NIAA-HSRC). Our project team has two lay members, as does our clinical reference group which provides support and advice on request. Patient representatives are full members of the study team and as such, are invited to comment on all aspects of continuing study development and implementation.

\section{Impact and dissemination}

A report of key findings will be produced annually. Quarterly study reports will also be disseminated to participating sites and published on the study web site. Research resulting from the study will be disseminated by presentations and publications in open-access peer-reviewed journals. Wider dissemination to various stakeholders, including the public, will be achieved via social media, podcasts, short videos, press releases, and written and electronic communications.

\section{Discussion}

The PQIP Patient study is the first national effort to report and improve patient outcomes from major surgery across multiple specialties other than mortality and length of stay. A number of research questions will be answered alongside the main purpose of quality improvement in perioperative patient care. The programme is notable in that it is being formally evaluated while in progress.

\section{Declarations}

\section{Ethics approval and consent to participate:}

The study has been approved by the Health Research Authority (HRA) as a research study, following review at the South-East Coast - Surrey Research Ethics Committee (REC reference: 16/LO/1827; IRAS project ID: 215928). All hospitals provide Caldicott Guardian approval in addition to confirmation of capacity and capability in accordance with usual HRA requirements.

\section{Consent for publication:}

not applicable

\section{Availability of data and materials:}

not applicable

\section{Acknowledgements:}


This manuscript is dedicated to the memory of our dear friend and colleague David Andrew Gilhooly.

\section{Author contributions:}

SRM: Study conception, design, statistical analysis plan, grant funding, delivery, project leadership. Manuscript drafting and revision.

DMcG: Study design and delivery; manuscript drafting.

PM: Statistical analysis plan; study design and delivery

All other authors: Study design and delivery, manuscript revision.

\section{All authors have approved the final manuscript.}

\section{Funding:}

This work is funded by the Royal College of Anaesthetists, the University College London Hospitals National Institute for Health Research Surgical Outcomes Research Centre and by the Health Foundation through an Improvement Science Fellowship for the Chief Investigator. PQIP has been adopted on to the NIHR portfolio of research studies in England \& Wales (CPMS ID 32256). POM-VLAD is supported by a Health Foundation Innovating for Improvement grant. ERAS Plus is supported by a Health Foundation Scaling Up Improvement Grant. SRM's employer receives reimbursement for her role as Director of the Health Services Research Centre. SRM was a Health Foundation Improvement Science Fellow between 2015 and 2019. This research was supported by the National Institute for Health Research (NIHR) Biomedical Research Centre based at University College London Hospitals NHS Foundation Trust. The views expressed are those of the authors and not necessarily those of the NHS, the NIHR or the Department of Health and Social Care.

\section{Competing interests:}

None

\section{References}

1. Abbott TEF, Fowler AJ, Dobbs TD, Harrison EM, Gillies MA, Pearse RM. Frequency of surgical treatment and related hospital procedures in the UK: a national ecological study using hospital episode statistics. Br J Anaesth. 2017;119:249-257.

2. Weiser TG, Haynes AB, Molina $G$ et al. Estimate of the global volume of surgery in 2012: an assessment supporting improved health outcomes. Lancet. 2015;385 Suppl 2:S11.

3. Pearse RM, Moreno RP, Bauer P et al. Mortality after surgery in Europe: a 7 day cohort study. Lancet. 2012;380:1059-1065. 
4. Ghaferi AA, Birkmeyer JD, Dimick JB. Complications, failure to rescue, and mortality with major inpatient surgery in medicare patients. Ann Surg. 2009;250:1029-1034.

5. Moonesinghe SR, Harris S, Mythen MG et al. Survival after postoperative morbidity: a longitudinal observational cohort study. Br J Anaesth. 2014;113:977-984.

6. Ghaferi AA, Birkmeyer JD, Dimick JB. Variation in hospital mortality associated with inpatient surgery. NEngl J Med. 2009;361:1368-1375.

7. Khuri SF, Henderson WG, DePalma RG, Mosca C, Healey NA, Kumbhani DJ. Determinants of longterm survival after major surgery and the adverse effect of postoperative complications. AnnSurg. 2005;242:326-341.

8. Toner A, Hamilton M. The long-term effects of postoperative complications. Curr Opin Crit Care. 2013;19:364-368.

9. Boney O, Moonesinghe SR, Myles PS, Grocott MP. Standardizing endpoints in perioperative research. Can J Anaesth. 2016;63:159-168.

10. Myles PS, Grocott MP, Boney O, Moonesinghe SR. Standardizing end points in perioperative trials: towards a core and extended outcome set. Br J Anaesth. 2016;116:586-589.

11. Skou ST, Roos EM, Laursen MB et al. A Randomized, Controlled Trial of Total Knee Replacement. N Engl J Med. 2015;373:1597-1606.

12. Kise NJ, Risberg MA, Stensrud S, Ranstam J, Engebretsen L, Roos EM. Exercise therapy versus arthroscopic partial meniscectomy for degenerative meniscal tear in middle aged patients: randomised controlled trial with two year follow-up. BMJ. 2016;354:i3740.

13. Allwood D. Engaging clinicians in quality improvement through national clinical audit. 2014. https://www.hqip.org.uk/resource/engaging-clinicians-in-qi-through-national-clinical-audit/. Accessed 10 May 2021.

14. Grocott MP, Browne JP, Van der Meulen J et al. The Postoperative Morbidity Survey was validated and used to describe morbidity after major surgery. J Clin Epidemiol. 2007;60:919-928.

15. Wong DJN, Oliver CM, Moonesinghe SR. Predicting postoperative morbidity in adult elective surgical patients using the Surgical Outcome Risk Tool (SORT). Br J Anaesth. 2017;119:95-105.

16. Dindo D, Demartines N, Clavien PA. Classification of surgical complications - A new proposal with evaluation in a cohort of 6336 patients and results of a survey. Annals of Surgery. 2004;240:205-213.

17. Silber JH, Williams SV, Krakauer H, Schwartz JS. Hospital and patient characteristics associated with death after surgery. A study of adverse occurrence and failure to rescue. Med Care. 1992;30:615-629.

18. Silber JH, Romano PS, Rosen AK, Wang Y, Even-Shoshan O, Holpp KG. Failure-to-rescue - Comparing definitions to measure quality of care. Medical Care. 2007;45:918-925.

19. Moonesinghe SR, Grocott MPW, Bennett-Guerrero E et al. American Society for Enhanced Recovery (ASER) and Perioperative Quality Initiative (POQI) joint consensus statement on measurement to maintain and improve quality of enhanced recovery pathways for elective colorectal surgery. Perioper Med (Lond). 2017;6:6. 
20. Moonesinghe SR, Jackson AIR, Boney $O$ et al. Systematic review and consensus definitions for the Standardised Endpoints in Perioperative Medicine initiative: patient-centred outcomes. $\mathrm{Br} \mathrm{J}$ Anaesth. 2019;123:664-670.

21. Faculty of Pain Medicine. Ask2Questions Screening Tool. https://fpm.ac.uk/standards-guidelinesinnovations/ask2questions. Accessed 10 May 2021.

22. Dawson J, Fitzpatrick R, Frost S, Gundle R, McLardy-Smith P, Murray D. Evidence for the validity of a patient-based instrument for assessment of outcome after revision hip replacement. J Bone Joint Surg Br. 2001;83:1125-1129.

23. Shulman MA, Myles PS, Chan MT, Mcllroy DR, Wallace S, Ponsford J. Measurement of disability-free survival after surgery. Anesthesiology. 2015;122:524-536.

24. Hutchings A, Neuburger J, Grosse Frie K, Black N, van der Meulen J. Factors associated with nonresponse in routine use of patient reported outcome measures after elective surgery in England. Health Qual Life Outcomes. 2012;10:34.

25. Hutchings A, Grosse Frie K, Neuburger J, van der Meulen J, Black N. Late response to patient-reported outcome questionnaires after surgery was associated with worse outcome. J Clin Epidemiol. 2013;66:218-225.

26. Hutchings A, Neuburger J, van der Meulen J, Black N. Estimating recruitment rates for routine use of patient reported outcome measures and the impact on provider comparisons. BMC Health Serv Res. 2014;14:66.

27. Neuburger J, Cromwell DA, Hutchings A, Black N, van der Meulen JH. Funnel plots for comparing provider performance based on patient-reported outcome measures. BMJ Qual Saf. 2011;20:10201026.

28. Neuburger J, Hutchings A, Allwood D, Black N, van der Meulen JH. Sociodemographic differences in the severity and duration of disease amongst patients undergoing hip or knee replacement surgery. $J$ Public Health (Oxf). 2012;34:421-429.

29. Neuburger J, Hutchings A, Black N, van der Meulen JH. Socioeconomic differences in patientreported outcomes after a hip or knee replacement in the English National Health Service. J Public Health (Oxf). 2013;35:115-124.

30. Neuburger J, Hutchings A, van der Meulen J, Black N. Using patient-reported outcomes (PROs) to compare the providers of surgery: does the choice of measure matter. Med Care. 2013;51:517-523.

31. Moonesinghe SR, Mythen MG, Das P, Rowan KM, Grocott MP. Risk stratification tools for predicting morbidity and mortality in adult patients undergoing major surgery: qualitative systematic review. Anesthesiology. 2013;119:959-981.

32. Protopapa KL, Simpson JC, Smith NC, Moonesinghe SR. Development and validation of the Surgical Outcome Risk Tool (SORT). Br J Surg. 2014;101:1774-1783.

33. Canet J, Gallart L, Gomar C et al. Prediction of postoperative pulmonary complications in a population-based surgical cohort. Anesthesiology. 2010;113:1338-1350. 
34. Chazapis M, Gilhooly D, Smith AF et al. Perioperative structure and process quality and safety indicators: a systematic review. Br J Anaesth. 2018;120:51-66.

35. Levy N, Mills P, Mythen M. Is the pursuit of DREAMing (drinking, eating and mobilising) the ultimate goal of anaesthesia? Anaesthesia. 2016;71:1008-1012.

36. Clavien PA, Barkun J, de Oliveira ML et al. The Clavien-Dindo classification of surgical complications: five-year experience. Ann Surg. 2009;250:187-196.

37. Bauer M, Bohrer H, Aichele G, Bach A, Martin E. Measuring patient satisfaction with anaesthesia: perioperative questionnaire versus standardised face-to-face interview. Acta Anaesthesiologica Scandinavica. 2001;45:65-72.

38. Walker EM, Bell M, Cook TM, Grocott MP, Moonesinghe SR, SNAP-1 IG. Patient reported outcome of adult perioperative anaesthesia in the United Kingdom: a cross-sectional observational study. $\mathrm{Br} \mathrm{J}$ Anaesth. 2016;117:758-766.

39. Stark PA, Myles PS, Burke JA. Development and psychometric evaluation of a postoperative quality of recovery score: the QoR-15. Anesthesiology. 2013;118:1332-1340.

40. Chazapis M, Walker EM, Rooms MA, Kamming D, Moonesinghe SR. Measuring quality of recovery-15 after day case surgery. $\mathrm{Br} J$ Anaesth. 2016;116:241-248.

41. Prytherch DR, Whiteley MS, Higgins B, Weaver PC, Prout WG, Powell SJ. POSSUM and Portsmouth POSSUM for predicting mortality. Physiological and Operative Severity Score for the enUmeration of Mortality and morbidity. Br J Surg. 1998;85:1217-1220.

42. Peden CJ, Moonesinghe SR. Measurement for improvement in anaesthesia and intensive care. $\mathrm{Br} \mathrm{J}$ Anaesth. 2016;117:145-148.

43. Deaton A, Cartwright N. Understanding and misunderstanding randomized controlled trials. Soc Sci Med. 2017

44. lacus SM, King G, Porro G. Causal Inference without Balance Checking: Coarsened Exact Matching. Polit Anal. 2012;20:1-24.

45. Bonfrer I, Figueroa JF, Zheng J, Orav EJ, Jha AK. Impact of Financial Incentives on Early and Late Adopters among US Hospitals: observational study. BMJ. 2018;360:j5622.

46. Moonesinghe SR, Wong DJN, Farmer L et al. SNAP-2 EPICCS: the second Sprint National Anaesthesia Project-EPIdemiology of Critical Care after Surgery: protocol for an international observational cohort study. BMJ Open. 2017;7:e017690.

47. NELA Project Team. First organisational report of the National Emergency Laparotomy Audit. 2014

48. May C. A rational model for assessing and evaluating complex interventions in health care. BMC Health Serv Res. 2006;6:86.

49. May C, Finch T, Mair F et al. Understanding the implementation of complex interventions in health care: the normalization process model. BMC Health Serv Res. 2007;7:148.

50. Atkins L, Francis J, Islam R et al. A guide to using the Theoretical Domains Framework of behaviour change to investigate implementation problems. Implement Sci. 2017;12:77. 
51. Pagel $\mathrm{C}$, Utley M, Crowe $S$ et al. Real time monitoring of risk-adjusted paediatric cardiac surgery outcomes using variable life-adjusted display: implementation in three UK centres. Heart. 2013;99:1445-1450.

52. Etzioni DA, Wasif N, Dueck AC et al. Association of hospital participation in a surgical outcomes monitoring program with inpatient complications and mortality. JAMA. 2015;313:505-511.

53. Moore JA, Conway DH, Thomas N, Cummings D, Atkinson D. Impact of a peri-operative quality improvement programme on postoperative pulmonary complications. Anaesthesia. 2017;72:317327. 